\title{
IDENTIFICATION OF BLUE-ALEURONE WHEAT DERIVATIVES BY SEQUENTIAL GISH-FISH ASSAY
}

\author{
Wang WEI ${ }^{1,2}$, Chen TIANQING ${ }^{2}$, Sui JIANSHU ${ }^{2}$, Wang YANLI ${ }^{2}$, Chai SHOUXI ${ }^{*}$, He QINGCAI \\ ${ }^{1}$ Gansu Agricultural University, Lanzhou, Gansu,P.R.CHINA \\ ${ }^{2}$ Guizhou Institute of Upland Crops, Guiyang, Guizhou,P.R.CHINA \\ *Corresponding author: sxchai@126.com
}

Received: 03.05.2017

\begin{abstract}
To select stable inheritable disomic substitution line from the filial generation of blue-grain wheat and common wheat high effectively, sequential GISH (genomic in situ hybridization) and multicolor FISH (fluorescence in situ hybridization) assay was used to investigate the number and characteristics of chromosome $4 \mathrm{E}$ in $\mathrm{F} 4$ and F6 hybrid offspring. The F4 and F6 plant were selected by the blue aleurone layer future, in which the bluegrain genes from Thinopyrum species were existed. GISH assay showed that the rate of stable inheritable disomic substitution line (blue-grain trait in common wheat) was above 80 percent in F6 plants, but lower in F4 plants. Specially, all of the F6 progenies of 'qian 09206', which was derived from crossing parents '0147' and common wheat '9938', were homozygous disomic substitution lines. The sequential GISH-FISH assay showed that $\mathrm{F} 4$ progeny (B-228) from filial generation of 'Neimai 10' and ' 0147 ' was a $4 \mathrm{D} / 4 \mathrm{E}$ stable inheritable alien disomic substitution line. These results showed that the color based selection was not effective. However, combining color based selection with sequential GISH-FISH assay can improve wheat breeding efficiency.
\end{abstract}

Key words: Blue-grained wheat, chromosome 4E, FISH, GISH

\section{INTRODUCTION}

The decaploid tall wheatgrass (Thinopyrum ponticum Liu \& Wang $=$ Agropyron elongatum, $2 \mathrm{n}=10 \mathrm{x}=70$, StStStStEeEeEbEbExEx) is perennial species that are related to wheat (Triticum aestivum L., $2 \mathrm{n}=42$ ) in the tribe Triticeae. It is long been known to have superior resistance to various diseases (e.g., leaf rust, powdery mildew, Wheat streak mosaic virus) and show environmental stresses resistance (e.g., cold, drought and salt stress). In addition, it has excellent properties such as multi-tillers, which is not exist in wheat. Thinopyrum ponticum can be easily crossed with wheat, making it a potent source of gene pool for wheat breeding (Li and Wang, 2009; Erkul et al, 2010). Blue-grained wheat lines were developed from Triticum Thinopyrum hybrid materials (Zheng et al., 2009). It is well known that the pigments in the blue-grained wheat are anthocyanin compounds deposited in the aleurone layer. Total anthocyanins in blue wheat averaged $157 \mathrm{mg} \mathrm{kg}-1$ in whole meal and $458 \mathrm{mg} \mathrm{kg}-1$ in bran (Abdel-Aal and Hucl, 1999). Anthocyanin pigments are helpful to scavenge free radical. In addition, Blue-grained wheat contains more free amino acids and vitamin, which is essential for human ( $\mathrm{Li}$ et al., 2004; Zong et al., 2006). In China, the first bluegrained wheat line, Blue 58, was developed from the wide cross between the common wheat variety Dami 953 and decaploid Th. ponticum, followed by successive backcrossing to wheat cultivars 573, Aifeng 3, and
Tienxuan 15 (Li et al., 1982). Cytogenetic analysis revealed that blue-grained gene(s) were derived from chromosome $4 \mathrm{E}$ of Thinopyrum ponticum (Zheng et al., 2009). Genetic analysis showed that many advanced agronomic traits, like stem diameter and blade width, were linked with bluegrained gene(s) (Zheng et al., 2009).

GISH (genomic in situ hybridization) is an effective means to identify alien chromosomes by labeling specific DNA sequence. It developed very fast after the nonradioactive DNA probes were used (Rayburn and Gill, 1985). Wu had selected new germplasm from the hybridization of Psathyrostachys huashanica and common wheat via GISH assistant technique (Wu et al., 2007). Li had used GISH to analysis wheat-Thinopyrum intermedium lines and identified Octoploid Tritelytrigia, disomic substitution lines and some addition lines (Li et al., 2016). More than the application in distant hybridization of wheat breeding, GISH has been used to answer a wide range of questions about genome relationships, origins of hybrid taxa and evolution (Chase et al., 2003).

Fluorescence in situ hybridization (FISH), which allows direct mapping of DNA sequences on chromosomes and fine distinguishing similar chromosomes, has become the most important technique in plant molecular cytogenetics research (Jiang and Gill, 2006). Presently, many sequence and corresponding probes had been tested or been 
commercialized. Given that the chromosome $4 \mathrm{E}$ of bluegrained wheat is small and display a similar shape with the chromosome 1D/4D/6D, multicolor FISH assay was used to precisely classify the chromosomes.

Given that blue-grain wheat shows no resistance to stripe rust and this disease often outbreak after pustulation period since the overcast and rainy weather in Guizhou province, 'blue 58' and common wheat '9988' (displaying resistance to stripe rust and powdery mildew) were chosen to integrate excellent agronomic traits and we had bred '0147' (qian green No.1). To integrate more excellent agronomic traits, like plant type, percentage of earringtillers and suitable maturity period, we further crossed ' 0147 ' with other germplasms with expected traits. The earlier selection was based on the color of aleurone layer, so it is hard to identify the chromosome details of progenies. Therefore, GISH and multicolor FISH were used to identify the chromosome details. After 5 times backcross and color based selection, most of the progenies contained stable alien 4E chromosomes.

\section{MATERIALS and METHODS}

\section{Germplasms and cross combination}

'0147' was developed from the hybridization of 'blue 58' and common wheat '9988'. It is a stable inheritable disomic substitution line, containing a pair of $4 \mathrm{E}$ chromosomes of Thinopyrum ponticum and displaying large panicle future and resistance to stripe rust and powdery mildew. Six kinds of common wheats were selected to cross with ' 0147 ' in 2009 or 2010 , followed by successive selfing several times. The no blue grains were excluded and F4 or F6 plants were analyzed. The common wheats, corresponding futures and the name of progenies crossed with ' 0147 'were list in table 1 . The rate of progenies, containing $4 \mathrm{E}$ chromosome, were analyzed in by GISH in different crossing combination. Sequential GISHFISH technique was used to finely describe the composition and futures of chromosomes in line 288 of F4 plants of 'Neimai 10/0147'.

Table 1. Cross combination between ' 0147 ' and common wheats.

\begin{tabular}{lll}
\hline Common wheats & Progenies & Generation system \\
\hline 9938 & qian 09197 & $\mathrm{~F} 6$ \\
Aibai & qian 09203 & $\mathrm{~F} 6$ \\
Aibai & qian 09206 & $\mathrm{~F} 6$ \\
9504 & $9504 / 0147$ & $\mathrm{~F} 4$ \\
MY2002 & $0147 / \mathrm{MY} 2002$ & $\mathrm{~F} 4$ \\
05 zhong27 & $0147 / 05 \mathrm{zhong} 27$ & $\mathrm{~F} 4$ \\
Neimai 10 & Neimai $10 / 0147$ & $\mathrm{~F} 4$ \\
\hline
\end{tabular}

\section{GISH and FISH}

Full and intact grains were selected and germinated at $23{ }^{\circ} \mathrm{C}$ in incubator. Root tip was cut for cytological assay when it was about $1-2 \mathrm{~cm}$ long. The preparation of metaphase chromosomes, DNA probes and GISH assay were carried out as Schwarzacher et al. described (Schwarzacher et al., 1992). Total genomic DNA from Thinopyrum ponticum was mechanically sheared and labeled with green fluorescein to get GISH probes. Total genomic DNA from $T$. aestivum cv. Chinese Spring was fragmented to pieces about $250 \mathrm{bp}$ long by autoclaving for 5 min and used as blocking DNA. The molar ratio is 300:1 between blocking DNA and probes. The sequence of probes used in FISH and the process were performed as Tang described (Tang et al., 2014). OLMPUS BX60 fluorescence microscope was used for observation and Cellsens Standard (Micropublisher 5.0; QImaging, Surrey, BC Canada) was used for image capture.

\section{RESULTS}

\section{The proportion of chromosome $4 E$ containing progenies in $\mathrm{F} 4$ plants}

Total 356 lines of F4 plant from different crossing combination were analyzed via GISH. All of the selected progenies were substitution line, containing 42 chromosomes, including one or two chromosome 4E. In detail, 291 lines were selected from Neimai 10/0147 plant, containing both disease resistance and nonresistance progenies. $59.4 \%$ of progenies contain one of chromosome $4 \mathrm{E}$ and $40.6 \%$ of progenies contain a pair of chromosome 4E. Further we have selected the plant, which showed resistance to powdery mildew, compact-type, multi-tillers, mezzo-maturing and plant high between $60-85 \mathrm{~cm}$, to analysis. Because these excellent properties are suitable for the environment of Guizhou province. 9, 14 and 17 lines were selected from the F4 plant of '0147/MY2002', '0147/05zhong27' and 'Neimai 10/0147', respectively. Data showed that two chromosome 4E containing progenies was more than $70 \%$ in all three kinds of crossing combination after 4 times selection (Table 2). Thus, the resistance based selection can improve the proportion of $4 \mathrm{E}$ chromosome after color based selection, proofing the linkage of blue gene(s) and resistance genes. Although color based selection could make sure of the existence of blue gene(s), it is not sure that if the plant is stable inheritable disomic substitution line.

\section{The proportion of chromosome $4 E$ containing progenies in F6 plants}

After 6 times of selection, the selected phenotypes were more stable than F4 plant. GISH assay was carried out in F6 plant of 'qian 09206', 'qian 09197' and 'qian 09203' respectively. Generally, more than $80 \%$ F6 plant contained a pair of 4E chromosome (Table 2). In detail, $81.8 \%$ of 'qian 09197 ', $88.9 \%$ of 'qian 09203 ' and $100 \%$ of 'qian 09206' plant was stable inheritable disomic substitution line and the remaining part contained one $4 \mathrm{E}$ chromosome, 
which was not stable inheritable. Representative GISH result was showed in figure 1. Therefore, GISH analysis is essential to accelerate the breeding stable inheritable germplasms, because color based selection could not exclude the unstable inheritable lines.

Table 2. Percentage of 4E chromosome containing progenies.

\begin{tabular}{lllll}
\hline Progenies & $\begin{array}{l}\text { Generation } \\
\text { system }\end{array}$ & Detected lines & $\begin{array}{l}\text { Single } \\
\text { chromosome 4E } \\
(\mathbf{\%})\end{array}$ & $\begin{array}{l}\text { Double } \\
\text { chromosome 4E } \\
(\mathbf{\%})\end{array}$ \\
\hline 9504/0147 & F4 & 9 & 22.2 & 77.8 \\
0147/MY2002 & F4 & 14 & 14.3 & 85.7 \\
0147/05zhong27 & F4 & 17 & 29.4 & 70.6 \\
Neimai 10/0147 & F4 & 291 & 59.4 & 40.6 \\
qian 09197 & F6 & 11 & 18.2 & 81.8 \\
qian 09203 & F6 & 9 & 11.1 & 88.9 \\
qian 09206 & F6 & 5 & 0 & 100 \\
\hline
\end{tabular}

\section{A1}

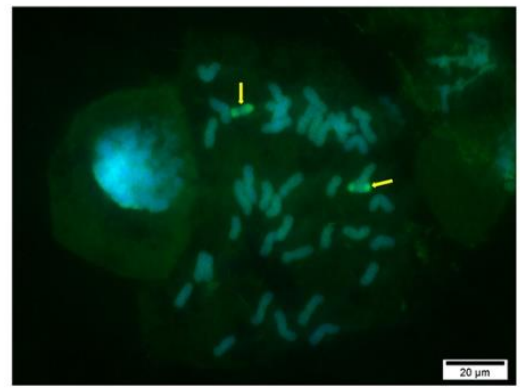

B1

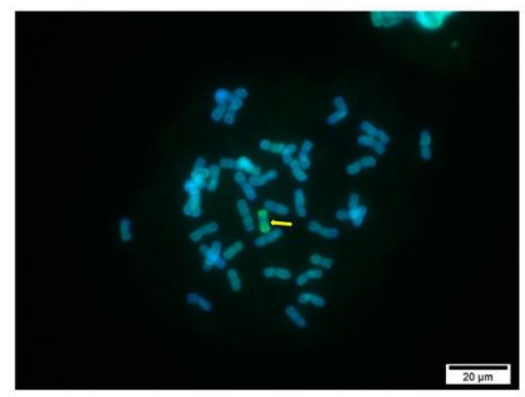

A2

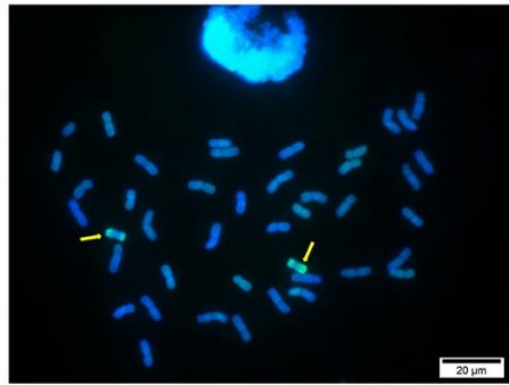

B2

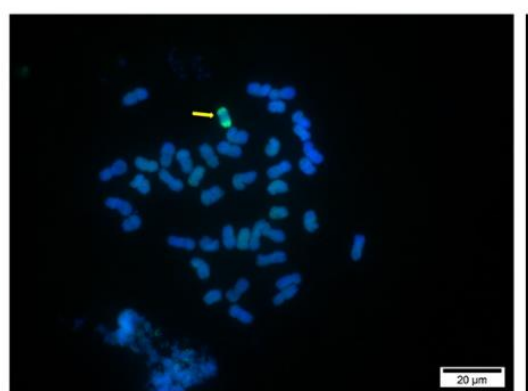

A3

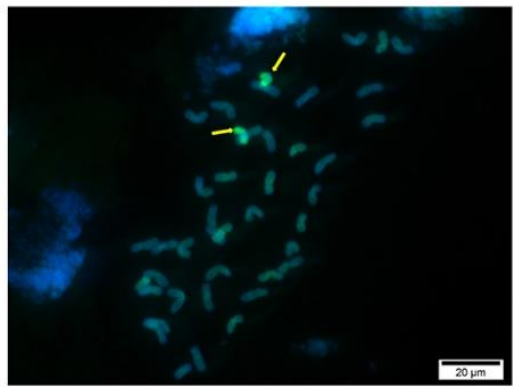

B3

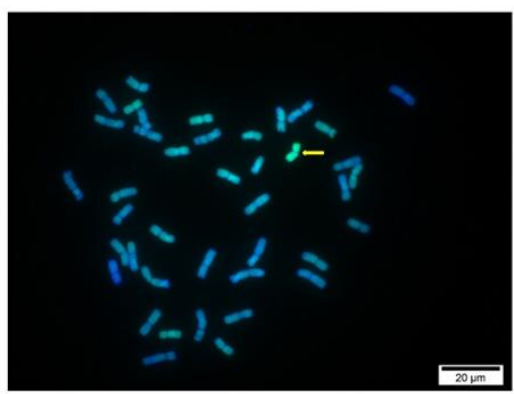

Figure 1. Representative GISH results of different Progenies. (A1-A3) containing two 4E chromosome. A1: qian 09203. A2 and A3: two lines of Neimai 10/0147. (B1-B3) containing one 4E chromosome. B1: 0147/05zhong27. B2 and B3: two lines of Neimai 10/0147. Green GISH probes can detect the DNA sequence of Thinopyrum ponticum. Back ground is Blue. Bars $=20 \mathrm{um}$.

\section{Sequential GISH-FISH analyze the composition of chromosomes in stable inheritable disomic substitution line}

To better understand the genetic background of derived germplasms, the line 228 in F4 plant of 'Neimai 10/0147' was selected to be analyzed by sequential GISH-FISH technique (Fig 2). After GISH assay, 42 chromosomes were detected and two 4E chromosomes were included. FISH assay showed that 4D chromosomes, being inherited from common wheat, could not be detected. Thus, we proposed that this derived germplasm was a 4D/4E stable inheritable alien disomic substitution line. 

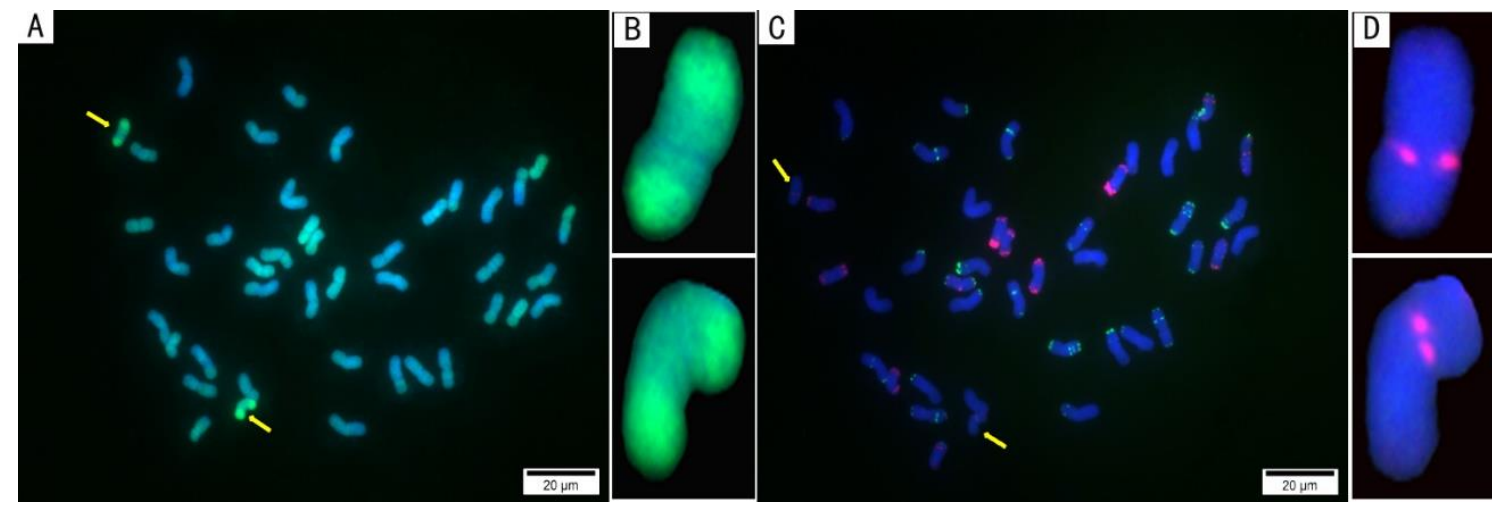

Figure 2. Sequential GISH-FISH results in blue-aleurone wheat derivatives (B-228). (A) GISH result by Thinopyrum ponticum derived green GISH probes. (B) Enlarged view of A. (C) FISH detection. Probe Oligo-pAs1-1 (red) and Oligo-pSc119.2-1 (green). (D) Enlarged view of C. 4E chromosomes were indicated by arrows and enlarged in B and D. Bars $=20$ um.

\section{DISCUSSION}

The first artificial hybridization between wheat and Thinopyrum spp. was made during the 1920 s -1930 s. Up to now, the wheat-Thinopyrum hybrids and resulting cultivars have made a great contribution to wheat production and genetic studies both in China and other parts of the world. The well know genes from tall or intermediate wheatgrasses are mainly those conferring resistance against leaf rust, stem rust, powdery mildew, barley yellow dwarf virus, wheat streak mosaic virus and wheat curl mite $(\mathrm{Li}$ and Wang, 2009). In addition, other important agronomic traits also can be introduced into wheat by distant hybridization, such as high efficiency of phosphorus absorption (Li et al., 1999), high photosynthetic efficiency (Peng et al., 2006) and high yield (Chen et al., 2013). However, it is unpractical that one variety performs everywhere. Based on the long time breeding experience in Guizhou province, we found that blue-grained wheat has wider leaf, thicker stem and more resistance to leaf rust but not resistance to stripe rust. Stripe rust disease often outbreak in Guizhou province. Therefore, to breed a stripe rust resistant variety is essential. ' 0147 ' is an alien disomic substitution line, in which 4D chromosomes was substituted by 4E chromosomes of 'blue 58'. '0147' performs good in Guizhou province but is not resistant to stripe rust. Many common wheat, showing stripe rust resistance, can be used as parent to cross with '0147'. So '9938', 'aibai', '9504', 'MY2002', '05zhong27' and 'Neimai 10' was chose. Because of the overcast and rainy weather after pustulation period of wheat in Guizhou province, photosynthetic efficiency is reduced, resulting in shriveled grain. It makes the color based selection more challenging. Our results showed that color based selection is an expensive and long process, which needs much work but does not distinguish homozygous and heterozygous $4 \mathrm{E}$ chromosome substitution line yet. Thus, we propose that color based selection is preferential at the beginning 2-3 generation (F2-F3) after hybridization, because the severe segregation at this time and just about $10 \%$ progenies contain all of selected traits. Selected agronomic traits are more stable and the disomic substitution line are more than
$70 \%$ in F4 plants. So, it is preferential to selected stable inheritable disomic substitution line by GISH at this time. It will be an effective method to breed new disomic substitution blue-grained wheat.

\section{ACKNOWLEDGEMENT}

This work was supported by National wheat industry technology system funding (CARS-3-2-43) and science and technology program of Guizhou province (NY[2013]3012).We are grateful to Dr. Zhang Shuqing, of the Guizhou university, for her modification and advices.

\section{LITERATURE CITED}

Abdel-Aal, E.S. M. and Hucl, P. 1999. A Rapid Method for Quantifying Total Anthocyanins in Blue Aleurone and Purple Pericarp Wheats. Cereal Chem. 76: 350-354

Chase, M.W., S. Knapp, A. Cox, J.J. Clarkson, Y. Butsko, J. Joseph, V. Savolainen and A. Parokonny. 2003. Molecular Systematics, GISH and the Origin of Hybrid Taxa in Nicotiana (Solanaceae). Ann. Bot. 92: 107-127.

Chen, S., Z. Huang, Y. Dai, S. Qin, Y. Gao, Y. Gao and J. Chen. 2013. Effect of E chromosomes of Thinopyrum elongatum on photosynthesis and yield characters in wheat. Journal of Yangzhou University (Agricultural and Life Science Edition). 1: 50-55.

Erkul, A., A. Ünay and C. Konak. 2010. Inheritance of Yield and Yield Components in a Bread Wheat (Triticum Aestivum L.) Cross. Turk. J. Field Crops. 15: 137-40.

Jiang, J. and Gill, B. S. 2006. Current status and the future of fluorescence in situ hybridization (FISH) in plant genome research. Genome. 49: 1057-1068.

Li, B-X., X-F. Zong and J-K. Zhang. 2004. Study on nutritional characters of wheat genotypes with different grain color. Journal of Southwest Agricultural University. 26: 753-755.

Li, H. and Wang, X. 2009. Thinopyrum ponticum and Th. intermedium: the promising source of resistance to fungal and viral diseases of wheat. J. Genet. Genomics. 36: 557-565.

Li, X-J., G. Li, T-Z. Hu, X-G. Hu, Y. Sun, Z-G. Ru, J. Song and L-L. Zhang. 2016. Agronomic traits and HMW-GS of 143 wheatThinopyrum intermedium derivatives and their GISH identification. Journal of Plant Genetic Resources. 17: 126-131.

Li, Y-J., J-Z. Liu, B. Li, J-Y. Li, S-J. Yao and Z-S. Li. 1999. Chromosomal control of the tolerance to phosphorus deficiency in genome of Triticum aestivum Chinese Spring. Acta Genet. Sin. 26: 529-538.

Li, Z-S., S-M. Mu, L-X. Jiang, H-P. Zhou, J-K. Wu and L. Yu. 1982. A Study on Blue-grained Monosomic Wheat (). Acta Genet. Sin. 6: 431439.

Peng, Y., Z. Peng, H. Song and J. Xu. 2006. Chromosomal Location of the Genes Associated with Photosynthesis of Lophopyrum elongatum 
(Host) A. Löve in Chinese Spring Background. Agricultural Sciences in China. 5: 579-586.

Rayburn, A. L. and Gill, B. S. 1985. Use of biotin-labeled probes to map specific DNA sequences on wheat chromosomes. J. Hered. 76: 78-81.

Schwarzacher, T., K. Anamthawat-Jónsson, G. E. Harrison, A. K. M. R. Islam, J. Z. Jia, I. P. King, A. R. Leitch, T. E. Miller, S. M. Reader, W. J. Rogers, M. Shi and J. S. Heslop-Harrison. 1992. Genomic in situ hybridization to identify alien chromosomes and chromosome segments in wheat. Theor. Appl. Genet. 84: 778-786.

Tang, Z., Z. Yang and S. Fu. 2014. Oligonucleotides replacing the roles of repetitive sequences pAs1, pSc119.2, pTa-535, pTa71, CCS1, and pAWRC.1 for FISH analysis. J. Appl. Genetics. 55: 313-318.
Wu, J., J-X Zhao, X-H Chen, S-H Liu, Q-H Yang, W-X Liu, F-Q Wei, J Dong and J-C Zhu. 2007. Cytology Characteristic and GISH Analysis on the Progenies Derived from Common Wheat (T. aestivum L.) $\times$ Psathyrostachys huashanica. J. Triticeae Crops. 27: 772-775.

Zong, X-F., J. Zhang and B. Li. 2006. Relationship between antioxidation and grain colors of wheat (Triticum aestivum L.). Acta Sci.-Agron. 32: 237-242.

Zheng, Q., B. Li, H-W. Li and Z-S. Li. 2009. Utilization of blue-grained character in wheat breeding derived from Thinopyrum poticum. J. Genet. Genomics. 36: 575-58 